\title{
Long-Term Evolution in Transit Duration of Extrasolar Planets from Magnetic Activity in their Parent Stars
}

\author{
Abraham Loeb \\ Astronomy Department, Harvard University, 60 Garden St., Cambridge, MA \\ 02138, USA
}

\begin{abstract}
Existing upper limits on variations in the photospheric radius of the Sun during the solar magnetic activity cycle are at a fractional amplitude of $\sim 2 \times 10^{-4}$. At that level, the transit duration of a close-in planet around a Sun-like star could change by a fraction of a second per year. This magnitude of variation is larger than that caused by other studied effects (owing to proper motion or general-relativistic effects), and should be included in the analysis of constraints on multi-planet systems from transit timing. A temporal correlation between the transit duration and spectroscopic measures of stellar activity can be used to separate the stellar radius change from other effects. The magnetic activity effect could be significantly larger for late-type stars, such as M-dwarfs, which are more variable than the Sun. In general, precision transit measurements provide a new tool for measuring long-term variations of stellar radii.
\end{abstract}

Key words: Stars: planetary systems, Sun: general;

PACS: 96.60.Bn; 97.82.Fs.

\section{Introduction}

Recently, it has been suggested that precise measurements of transit timing variations can reveal the existence of additional planets in known planetary systems (Miralda-Escudé 2002; Agol et al. 2005; Holman \& Murray 2005; Heyl \& Gladman 2006). Related observations are already placing interesting constraints on planetary companions (Steffen \& Agol 2005; Agol \& Steffen 2005; Ribas et al. 2008; Miller-Ricci et al. 2008a), despite the intrinsic noise

Email address: aloeb@cfa.harvard.edu (Abraham Loeb). 
introduced by stellar activity (Miller-Ricci et al. 2008b). Space missions, such as $\operatorname{MOST}^{1}$, COROT $^{2}$ and Kepler 3 , are capable of detecting timing variations over their lifetime at a sensitivity better than a second per year (Pál \& Kocsis 2008; Jordán \& Bakos 2008; Rafikov 2008).

Aside from the dynamical interaction of the transiting planet or parent star with another object, it was recognized that general relativistic effects (Pál \& Kocsis 2008; Jordán \& Bakos 2008) as well as the proper motion of the system (Rafikov 2008), would introduce variations in the transit timing and duration at levels smaller than a second per year. Here, we point out that the magnetic activity cycle of the host star may cause long-term variations in the stellar radius on timescales of years that could make an even larger contribution to the observed transit duration variations. In $\S 2$ we calibrate the magnitude of this effect based on existing data for the Sun. Finally, in $\S 3$ we comment on the implications of future transit measurements of these magnetic activity variations in other stars.

\section{Method}

The transit duration of a planet on a circular orbit of radius $a$ around a star of mass $M_{\star}$ and radius $R_{\star}$ is given by,

$$
t_{\text {tran }}=2 \frac{R_{\star}\left(1-p^{2}\right)^{1 / 2}}{\left(G M_{\star} / a\right)^{1 / 2}},
$$

where $p$ is the minimum separation between the planetary trajectory and the stellar disk center on the sky, in units of $R_{\star}$. The fractional change in the transit duration timing (TDV) owing to a small fractional variation in the stellar radiuk $4, \Delta_{R_{\star}}$, is given for $\Delta_{R_{\star}} \ll\left[\left(1-p^{2}\right) / 2 p^{2}\right]$ by

$$
\begin{aligned}
& \Delta t_{\text {tran }}=\Delta_{R_{\star}} t_{\operatorname{tran}}=\left(1-p^{2}\right)^{1 / 2} \times \\
& \times 1.49 \mathrm{~s}\left(\frac{\Delta_{R_{\star}}}{10^{-4}}\right)\left(\frac{M_{\star}}{M_{\odot}}\right)^{-1 / 2}\left(\frac{R_{\star}}{R_{\odot}}\right)\left(\frac{a}{0.1 \mathrm{AU}}\right)^{1 / 2},
\end{aligned}
$$

\footnotetext{
$\overline{1 \text { http://www.astro.ubc.ca/MOST/ }}$

2 http://smsc.cnes.fr/COROT/

3 http://kepler.nasa.gov/

4 A change in the image shape of the parent star could change the transit timing in a more complicated way. Limits on fractional variations in the oblateness of the Sun during the solar cycle are at the level of $\sim 10^{-5}$ (Kuhn et al. 1998).
} 
where $\Delta_{R_{\star}} \equiv\left(\Delta R_{\star} / R_{\star}\right)$. For $\Delta_{R_{\star}} \gg\left[\left(1-p^{2}\right) / 2 p^{2}\right]$, the transit duration could either diminish as the stellar image shrinks or increase by $\Delta t_{\text {tran }}=$ $\left[2 p^{2} \Delta_{R_{\star}} /\left(1-p^{2}\right)\right]^{1 / 2} t_{\text {tran }}$ as the image grows, but we regard this grazingincidence regime as rare and unlikely.

The solar magnetic activity cycle is known to cause fractional changes of $\sim 0.1 \%$ in the distribution of the near-UV, visible, and near-IR brightness of the Sun relative to the ecliptic plane between solar minimum and maximum (Fröhlich 2006; Foukal \& Bernasconi 2008). At solar maximum, cold spots cover a larger fraction of the surface area of the Sun, but the surface brightness in between the spots increases due to energy deposition by magnetic activity. Helioseismological data implies variations in the rotational kinetic energy at a fractional level of $\sim 0.2 \%$ (Antia, Chitre, \& Gough 2008), as well as in the sound speed at the base of the convective zone at a fractional level of $\left(\Delta c^{2} / c^{2}\right) \sim 0.7 \times 10^{-4}$ (Baldner \& Basu 2008), over the solar cycle. The associated change in the pressure supporting the gas would inevitably produce a variation in the physical radius of the star. In the context of TDV, we are interested in the variation of the apparent size of the photospheric stellar disk on the sky, where the optical depth to emergent radiation is of order unity.

State-of-the-art studies of the solar radius place an upper limit $\Delta_{R_{\odot}}<2 \times 10^{-4}$ on its fractional variation over periods of decades (Djafer, Thuillier, \& Sofia 2008; Lefebvre et al. 2006; Kuhn et al. 2004). Reports on the detection of a variable solar radius have been controversial (e.g., Noel 2004, Lefebvre \& Kosovichev 2005). Helioseismological data implies a $\sim 10^{-4}$ change in the sound speed at the base of the convective zone (Baldner \& Basu 2008). However, the fractional variation in the solar radius could be much smaller (see also Stothers 2006). Nevertheless, it is interesting to examine the sensitivity of future transit timing measurements to variations of $\Delta_{R_{\odot}} \sim 10^{-4}$ over the solar cycle. Substituting this (upper limit) value in equation (2) leads to a TDV at a level of $\sim 0.3(a / 0.1 \mathrm{AU})^{1 / 2}$ seconds per year over a cycle of $\sim 5.5$ years in transits of close-in planets around Sun-like stars 5 . Interestingly, these variations are larger than other effects which have been considered in the literature as detectable (Pál \& Kocsis 2008; Jordán \& Bakos 2008; Rafikov 2008), and so we conclude that a search for TDV would provide interesting new limits on stellar radius variations that are competitive with state-of-the-art measurements for the Sun. A generic signature of the TDV induced by a circularly symmetric variation in stellar radius is that the timing of the transit center remains unchanged as the transit duration changes.

\footnotetext{
5 Shorter-term variations with a higher amplitude (flares) introduce noise for TDV
} in all stellar types. 


\section{Discussion}

Precision timing of planetary transits offers a new tool for measuring long-term variations in stellar radii. Such measurements would provide unprecedented constraints on the surface conditions during the magnetic activity cycles of stars at levels that are not accessible for the Sun. In difference from rare transits within the solar system, the short orbital period of close-in planets around other stars and the use of the cumulative flux from the stellar disk offer improved prospects for averaging over fluctuations in the stellar limb (owing to surface spots or other phenomena).

The magnetic activity origin of the TDV can be revealed through its temporal cross-correlation with stellar brightness variations (in analogy to the Sun; see Frölich 2006) and with spectral indicators such as the Ca K line (Baliunas 2006). A temporal correlation of this type can, for example, help to distinguish between the effect of a stellar radius change and a change in the inclination of the planet's orbit. In general, the magnetic cycle effect should be kept in mind when inferring dynamical constraints on multi-planet systems or on proper motion from transit timing.

We emphasize that the TDV induced by magnetic activity could be significantly larger for late-type stars, such as M-dwarfs, which are more variable than the Sun (Schmidt et al. 2007; Lane et al. 2007; Rockenfeller et al.2006).

\section{Acknowledgments}

I thank Dan Fabrycky and John Raymond for helpful discussions. This work was supported by in part by NASA grant NNX08AL43G, by FQXi, and by Harvard University funds.

\section{References}

Agol, E., Steffen, J., Sari, R., \& Clarkson, W. 2005, MNRAS, 359, 567

Agol, E., \& Steffen, J. H. 2007, MNRAS, 374, 941

Antia, H. M., Chitre, S. M., \& Gough, D. O. 2008, A \& A, 477, 657

Baldner, C. S., \& Basu, S. 2008, ArXiv e-prints, 807, arXiv:0807.0442

Baliunas, S. L. 2006, Solar and Stellar Activity Cycles, 26th meeting of the IAU, Joint Discussion 8, 17-18 August 2006, Prague, Czech Republic, JD08, \#65, 8,

Djafer, D., Thuillier, G., \& Sofia, S. 2008, ApJ, 676, 651 
Foukal, P., \& Bernasconi, P. N. 2008, Solar Phys., 248, 1

Fröhlich, C. 2006, Space Science Reviews, 125, 53

Heyl, J. S., \& Gladman, B. J. 2007, MNRAS, 377, 1511

Holman, M. J., \& Murray, N. W. 2005, Science, 307, 1288

Jordán, A., \& Bakos, G. A. 2008, ArXiv e-prints, 806, arXiv:0806.0630

Kuhn, J. R., Bush, R. I., Scherrer, P., \& Scheick, X. 1998, Nature, 392, 155

Kuhn, J. R., Bush, R. I., Emilio, M., \& Scherrer, P. H. 2004, ApJ, 613, 1241

Lane, C., et al. 2007, ApJL, 668, L163

Lefebvre, S., \& Kosovichev, A. G. 2005, ApJL, 633, L149

Lefebvre, S., Bertello, L., Ulrich, R. K., Boyden, J. E., \& Rozelot, J. P. 2006, ApJ, 649,444

Miller-Ricci, E., et al. 2008, ArXiv e-prints, 802, arXiv:0802.0718

Miller-Ricci, E., et al. 2008, ArXiv e-prints, 802, arXiv:0802.2722

Miralda-Escudé, J. 2002, ApJ, 564, 1019

Noël, F. 2004, A \& A, 413, 725

Pál, A., \& Kocsis, B. 2008, ArXiv e-prints, 806, arXiv:0806.0629

Rafikov, R. R. 2008, ArXiv e-prints, 807, arXiv:0807.0008

Ribas, I., Font-Ribera, A., \& Beaulieu, J.-P. 2008, ApJL, 677, L59

Rockenfeller, B., Bailer-Jones, C. A. L., \& Mundt, R. 2006, A \& A, 448, 111

Schmidt, S. J., Cruz, K. L., Bongiorno, B. J., Liebert, J., \& Reid, I. N. 2007, AJ, 133,2258

Steffen, J. H., \& Agol, E. 2005, MNRAS, 364, L96

Stothers, R. B. 2006, ApJL, 653, L73 